Schmerz 2016 $30: 493-495$

DOI 10.1007/s00482-016-0170-1

Online publiziert: 26 . Oktober 2016

(c) Deutsche Schmerzgesellschaft e.V. Published by Springer-Verlag Berlin Heidelberg - all rights reserved 2016

CrossMark

\author{
L. Radbruch ${ }^{1,2} \cdot$ M. Schäfer ${ }^{3,4}$ \\ 'Klinik für Palliativmedizin, Universitätsklinikum Bonn, Bonn, Deutschland \\ ${ }^{2}$ Deutsche Gesellschaft für Palliativmedizin, Berlin, Deutschland \\ ${ }^{3}$ Klinik für Anästhesiologie und operative Intensivmedizin, Charité - Universitätsmedizin, Campus \\ Virchow, Berlin, Deutschland \\ ${ }^{4}$ Deutsche Schmerzgesellschaft, Berlin, Deutschland
}

\title{
Tumorschmerz
}

\section{Neue Perspektiven}

Die überwiegende Mehrzahl von Tumorpatienten wird im Verlauf der malignen Erkrankung unter behandlungsbedürftigen Schmerzen leiden. Glücklicherweise verfügen wir über wirksame Methoden zur Schmerzlinderung, in erster Linie durch die systemische Behandlung mit Opioiden. Der Stellenwert von Morphin und anderen Opioiden in der Tumorschmerztherapie wurde in der Leitlinie der Weltgesundheitsorganisation vor nunmehr 30 Jahren festgeschrieben [11] und in späteren Leitlinien immer wieder bestätigt. Die Leitlinien der European Association for Palliative Care (EAPC) haben vor wenigen Jahren diesen Stellenwert evidenzbasiert bestätigt [1].

Die Segnungen der Linderung von Tumorschmerzen mit Opioiden, wie sie in Deutschland und anderen europäischen Ländern mittlerweile in allen Bereichen der Onkologie angewandt wird, werden äußerst deutlich in den Berichten aus Entwicklungsländern, in denen diese Behandlungsmöglichkeit nicht oder nur sehr eingeschränkt gegeben ist. Dokumentarfilme wie „Life before Death" (www.lifebeforedeath. com) ebenso wie Publikationen der internationalen Fachgesellschaften $[2,3]$ und der Menschenrechtsorganisation Human Rights Watch $[7,8]$ zeigen ein erschreckendes Bild, wie Menschen mit Tumorerkrankung und ohne Zugang zu Opioiden leiden müssen.

In der vorliegenden Ausgabe von Der Schmerz sind nun gleich zwei Beiträge, die ein anderes Licht auf die Tumorschmerztherapie werfen.

\section{Was leisten die psycho- edukativen Interventionen?}

Kühne et al. werten in ihrer systematischen Übersichtsarbeit 32 kontrollierte Studien zu schmerzpsychologischen Interventionen bei Tumorpatienten aus. In der Mehrzahl wurden psychoedukative Interventionen getestet, und die Mehrzahl der Studien bei Patientinnen mit Mammakarzinom und in frühen Erkrankungsstadien durchgeführt.

Die Autoren beklagen, dass die Interventionen in den Studien oft durch Pflegepersonal ohne spezielle Schmerzausbildung im ambulanten Setting als Kurzintervention erfolgten. Die Autoren stellen heraus, dass die Therapieergebnisse in den klinischen Studien besser wären, wenn die Therapeuten eine fundierte Ausbildung wie die Spezielle Schmerzpsychotherapie (die von den vier führenden deutschen Schmerzfachgesellschaften Deutsche Schmerzgesellschaft, DGPSF, DMKG und DGS entwickelt wurde) gehabt hätten.

Allerdings ist aus unserer Sicht infrage zu stellen, ob alle Patienten eine Intervention durch spezialisierte Schmerzpsychotherapeuten benötigen. Ähnlich wie bei der abgestuften allgemeinen und spezialisierten Palliativversorgung sollten auch die psychotherapeutischen Interventionen für Tumorpatienten abgestuft angeboten werden. Jeder Onkologe und jeder Palliativmediziner sollte entsprechende Grundkenntnisse haben, Ärzte und Pflegekräfte mit entsprechender Qualifikation sollten in ihrer Ausbildung psychotherapeutische Verfahren zur Schmerzlinde- rung erlernt haben und niedrigschwellig anwenden können, also zum Beispiel in der häuslichen Umgebung, ohne dass der Patient dafür die Klinik aufsuchen muss. Das gilt besonders für die psychoedukativen Verfahren, während zum Beispiel Hypnose oder verhaltenstherapeutische Verfahren eher den Spezialisten überlassen bleiben sollten.

Kühne et al. leisten allerdings einen wichtigen Beitrag, weil sie darauf hinweisen, dass die medikamentöse Tumorschmerztherapie nur einen Teil der Schmerzbehandlung darstellt und bei vielen Patienten ergänzt werden muss zu einem multimodalen Therapiekonzept. Kühne et al. beklagen, dass diese Einbindung in den untersuchten Studien nur ungenügend ist, und schlagen vor, die schmerzbedingte Beeinträchtigung („disability") vermehrt als Endpunkt in Studien zur Tumorschmerztherapie zu nutzen.

\section{Opioideinsatz bei „Tumorüberlebenden"}

Auch Wirz et al. versuchen, eine neue Perspektive auf den Tumorschmerz zu liefern, mit ihrer Übersichtsarbeit, die allerdings nicht auf einer systematischen Literatursuche basiert. Die Autoren haben vor allem die mit den Fortschritten in der Onkologie zunehmende Zahl von Überlebenden, die unter chronisch andauernden Schmerzen leiden, im Blick. Sie postulieren, dass bei Tumorpatienten mit persistierenden Schmerzen nach und trotz kurativer Tumorbehandlung die gleichen Chronifizierungsmechanismen 
wie bei anderen chronischen Schmerzen auftreten und auch die gleichen Risikofaktoren für Abhängigkeiten bestehen. Sie fordern für diese Tumorüberlebenden eine multimodale Therapie und die Einführung eines Screenings für das Abhängigkeitsrisiko.

Diese Arbeit zeigt allerdings auch die Fallstricke in der Betrachtung, nämlich Patienten, die nach erfolgreicher Behandlung als geheilt gelten, Patienten mit chronischen Schmerzen bei langem Krankheitsverlauf (zum Beispiel Mammakarzinom) und Patienten mit Tumorschmerz insgesamt zu vermischen. Hier ist aber eine differenzierte Betrachtung notwendig.

Niemand wird doch ernsthaft vorschlagen, einem Patienten mit Zungengrundkarzinom und Alkoholmissbrauch in der Vergangenheit eine Opioidtherapie zu verweigern, weil sein Abhängigkeitsrisiko zu hoch sei. Im Gegenteil, in der Palliativmedizin werden aktuell Standards für die Tumorschmerztherapie bei $\mathrm{Pa}$ tienten in Substitutionsbehandlung entwickelt - nicht weil diese Patienten ein Suchtrisiko haben, sondern weil die Organisation der Versorgung kompliziert ist, wenn zum Beispiel tägliche Besuche in der Substitutionsambulanz notwendig sind.

Auf der anderen Seite ist es wahrscheinlich sinnvoll, die Überlebenden einer Tumorerkrankung eher nach den Richtlinien für chronische nichttumorbedingte Schmerzen zu behandeln. In der Expertengruppe, die vor Kurzem von der Weltgesundheitsorganisation zur Überarbeitung der WHO-Empfehlungen zur Tumorschmerztherapie einberufen wurde, wurde tatsächlich als erste wichtige Überlegung beschlossen, die zu entwickelnde Leitlinie auf Patienten mit aktiver Tumorerkrankung zu beschränken, also Überlebende nicht in dieser Leitlinie zu berücksichtigen.

Wirz et al. diskutieren ausführlich das Risiko einer Abhängigkeit bei Opioidtherapie. Hauptzeuge der Anklage ist hier Jane Ballantyne, die als Vorsitzende von Physicians for Responsible Opioid Prescription (PROP) für einen möglichst restriktiven Opioidgebrauch kämpft. Sie sieht in der zunehmenden Zahl von Opioidverordnungen (der Opioidepide- mie) in den USA den Hauptgrund für die gleichzeitig zunehmende Zahl von Opioidabhängigen und von Drogentoten mit Opioidgebrauch. Allerdings ist nicht klar, wie häufig hierbei der (illegale) Gebrauch von verordneten Opioiden durch Patienten ohne Schmerzen, die (versehentliche) Überdosierung von Patienten mit chronischen Schmerzen oder ein erhöhtes Mortalitätsrisiko bei bestimmungsgemäßem Gebrauch einer durch den Arzt verordneten Opioidtherapie beteiligt ist. Interessanterweise lag laut einem Kassenbericht einer großen deutschen Krankenkasse [9] die 1-JahresPrävalenz für Opioidmissbrauch bzw. Sucht in Deutschland bei 0,008 \% (d.h. 8 von 100.000), trotz stetig zunehmender Verschreibungen von Opioiden in den letzten zehn Jahren [10], was zeigt, dass die Situation in Deutschland und den USA nicht vergleichbar ist.

\section{Paradigmenwechsel in den USA}

Auf dem World Congress on Pain stellte Jane Ballantyne vor wenigen Tagen vor, dass die "alten Lehrmeinungen“ in den USA („Opioide sind nicht gefährlich und machen nicht abhängig"), die sie vor allem dem in der Vergangenheit exponentiell gesteigerten Pharma-Marketing und dessen unkritischer Übernahme durch Ärzte zuschreibt, durch neue Lehrmeinungen ersetzt werden müssen („Opioide sind sehr gefährlich und können leicht zur Abhängigkeit führen“).

Die neuen Leitlinien zur Opioidtherapie der Centers for Disease Control [4] greifen diese Position auf und warnen vor den Gefahren. Allerdings werden Schmerzen bei aktiver Tumorerkrankung, in der Palliativversorgung und am Lebensende bei diesen Empfehlungen ausdrücklich ausgenommen!

Die Warnung vor dem Abhängigkeitsrisiko bei einer Opioidtherapie bei Tumorpatienten ist umstritten. Ein nennenswertes Risiko entspricht jedenfalls nicht unseren Erfahrungen. Einer der beiden Autoren (L. Radbruch) hat persönliche Erfahrungen aus der Zeit der Publikation von Zech et al. [12] zur Behandlung von mehr als 2000 Patienten nach den WHO-Empfehlungen, in dieser Kohorte wurde kein Problem mit Opioidabhängigkeit gefunden. Steigerungen der Opioiddosis werden bei Tumorpatienten fast immer durch den Progress der Grunderkrankung erklärt, nicht durch Toleranzentwicklung. Die benötigten Dosierungen sind oft viel höher als die definierten Tagesdosierungen (bei oralem Morphin $100 \mathrm{mg}$ ), aber dies steht im Zusammenhang mit dem überwältigenden nozizeptiven Input durch den Tumor. Vor allem aber: Unter einer sachgerechten Opioidtherapie verspüren die Tumorpatienten keine nachteiligen Folgen, weder körperlich noch sozial. Sie sind im Gegenteil wieder in der Lage, ihr Leben zu genießen, Aktivitäten auszuführen, sozial zu interagieren, weil sie jetzt nicht mehr durch die Tumorschmerzen daran gehindert werden. Die Opioidepidemie scheint vor allem ein US-amerikanisches Problem zu sein, denn in den europäischen Ländern wird kein solcher Anstieg von Überdosierungen und Todesfällen berichtet [6]. Dies kann eine Vielzahl von Gründen aufseiten von Patienten, Ärzten und der Gesellschaft haben. Unter anderem wird der vorsichtigere Umgang mit Opioiden bei nichttumorbedingten Schmerzen in der S3-Leitlinie [5] ausgedrückt, in der Indikationen für ein selektives Patientengut, klare Kontraindikationen und die Notwendigkeit der regelmäßigen Reevaluation und Risiko/Nutzen-Bewertung für die Langzeitopioidtherapie benannt werden. Somit erfordert die sichere und wirksame Anwendung von Opioiden in der medizinischen Versorgung die Herausforderung, beide Seiten dieser Medaille anzunehmen.

Im Gegenzug besteht durchaus die Gefahr, dass die Warnungen vor der Opioidtherapie dazu führen, dass Tumorpatienten trotz entsprechender Indikation der Zugang zu solchen Medikamenten erschwert wird und sie damit unnötig leiden müssen.

Den Autoren gebührt Dank, dass sie diese Perspektiven vorlegen und damit eine Diskussion ermöglichen. Tatsächlich ist Tumor nicht gleich Tumor. Überlebende mit postoperativem Schmerz oder Schmerz nach Strahlentherapie sollten eher nach den Empfehlungen für chronischen nichttumorbedingten Schmerz behandelt werden. Bei Pati- 
enten mit aktiver Tumorerkrankung ist zumindest die Kenntnis der von Wirz et al. aufgeführten Zeichen der Abhängigkeit notwendig. Allerdings sollte ein Abhängigkeitsrisiko nicht dazu führen, dass Patienten zum Beispiel trotz rasch progredienter Erkrankung kein Opioid erhalten. Mögliche Bedenken sollten nicht die effektive Opioidtherapie behindern.

Immer aber sollte die Tumorschmerztherapie in ein patientenbezogenes individuelles Therapiekonzept eingebunden werden. Dies kann wie in der Palliativversorgung auf der körperlichen, psychischen, sozialen und spirituellen Ebene oder in der Schmerztherapie im biopsychosozialen Modell erfolgen.
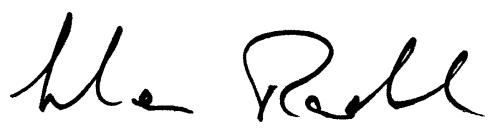

L. Radbruch

\section{Korrespondenzadresse}

\section{Prof. Dr. L. Radbruch}

Klinik für Palliativmedizin, Universitätsklinikum Bonn

Sigmund-Freud-Str. 25, 53127 Bonn,

Deutschland

Lukas.Radbruch@malteser.org

Interessenkonflikt. L. Radbruch und M. Schäfer geben an, dass kein Interessenkonflikt besteht.

\section{Literatur}

1. Caraceni A, Hanks G, Kaasa S, Bennett MI, Brunelli C, Cherny N, Dale O, De Conno F, Fallon M, Hanna $M$, Haugen DF, Juhl G, King S, Klepstad P, Laugsand EA, Maltoni M, Mercadante S, Nabal M, Pigni A, Radbruch L, Reid C, Sjogren P, Stone PC, Tassinari D, Zeppetella G (2012) Use of opioid analgesics in the treatment of cancer pain: evidence-based recommendations from the EAPC. Lancet Oncol 13(2):e58-e68

2. Cherny NI, Cleary J, Scholten W, Radbruch L, Torode J (2013) The Global Opioid Policy Initiative (GOPI) project to evaluate the availability and accessibility of opioids for the management of cancer pain in Africa, Asia, Latin America and the Caribbean, and the Middle East: introduction and methodology. Ann Oncol 24(Suppl 11):xi7-xi13

3. De Lima L, Pastrana T, Radbruch L, Wenk R (2014) Cross-sectional pilot study to monitor the availability, dispensed prices and affordability of opioids around the globe. J Pain Symptom Manage 48(4):649-659.e1. doi:10.1016/j.jpainsymman. 2013.12.237

4. Dowell D, Haegerich TM, Chou R (2016) CDC guideline for prescribing opioids for chronic pain. MMWR Recomm Rep 65:1-49
5. Hauser W, Bock F, Engeser P, Hege-Scheuing G, Huppe M, Lindena G, Maier C, Norda H, Radbruch L, Sabatowski R, Schafer M, Schiltenwolf M, Schuler M, Sorgatz H, Tolle T, Willweber-Strumpf A, Petzke $F$ (2015) Empfehlungen der aktualisierten Leitlinie LONTS : Langzeitanwendung von Opioiden bei chronischen nicht-tumorbedingten Schmerzen. Schmerz 29(1):109-130

6. Hauser W, Petzke F, Radbruch L, Tolle TR (2016) The opioid epidemic and the long-term opioid therapy for chronic noncancer pain revisited:a transatlantic perspective. Pain Manag 6(3):249-263

7. Human Rights Watch (2009) „Please, do not make us suffer any more..." Access to pain treatment as a human right. Human Rights Watch, New York

8. Human Rights Watch (2013) Abandoned in agony - cancer and the struggle for pain treatment in Senegal. http://www.hrw.org/print/reports/ 2013/10/24/abandoned-agony. Zugegriffen: 1. Dezember 2013
9. Marschall U, L'Hoest H, Radbruch L, Hauser W (2016) Long-term opioid therapy for chronic noncancer pain in Germany. Eur JPain 20(5):767-776

10. Schubert I, Ihle P, Sabatowski R (2013) Increase in opiate prescription in Germany between 2000 and 2010: a study based on insurance data. Dtsch Arztebl Int 110(4):45-51

11. World Health Organisation (1986) Cancer pain relief. World Health Organisation, Genf

12. Zech DF, Grond S, Lynch J, Hertel D, Lehmann KA (1995) Validation of World Health Organization Guidelines for cancer pain relief: a 10-year prospective study. Pain 63:65-76

\section{Alle Inhalte von springermedizin.de online im Volltext lesen}

Mit e.Med Interdisziplinär - dem Kombi-Abo von SpringerMedizin.de - können Sie jederzeit auf alle Inhalte zugreifen, die Sie als Arzt für Ihren Praxis- oder Klinikalltag benötigen. Sie sind immer aktuell informiert - über die neuesten Publikationen, wichtige Studien oder innovative Therapieverfahren.

Die Vorteile von e.Med Interdisziplinär:

- Gestalten Sie Ihre Fortbildung nach Ihren Bedürfnissen:

e.Med Interdisziplinär bietet Ihnen Online-Zugang zu 600 CME-Fortbildungskursen aller Fachrichtungen

- Sie entscheiden, was Sie lesen möchten:

Alle Fachzeitschriften stehen digital im Volltext zur Verfügung.

- Finden Sie die gewünschten Informationen auch in englischen Publikationen:

Sie können in mehr als 500 englischsprachigen Fachzeitschriften online

recherchieren und auf die Suchergebnisse uneingeschränkt im Volltext zugreifen.

- Auf Wunsch erhalten sie darüber hinaus eine gedruckte Fachzeitschrift nach Wahl.

NEU: Mit den fachspezifischen e.Med-Abos können Sie sich effizient innerhalb Ihres Fachgebietes auf dem Laufenden halten. Das Angebot reicht von AINS bis Radiologie - Sie haben die Wahl!

Testen Sie e.Med 30 Tage kostenlos und unverbindlich!

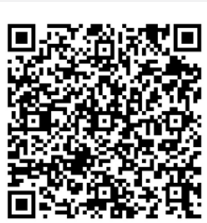

Jetzt informieren unter www.springermedizin.de/eMed oder telefonisch unter 0800-77 80777 (Montag bis Freitag, 10 bis 17 Uhr) 